\title{
Replication of genetic association studies in asthma and related phenotypes
}

\author{
Siizkhuu Undarmaa ${ }^{1}$, Yoichi Mashimo ${ }^{1}$, Satoshi Hattori ${ }^{1}$, Naoki Shimojo ${ }^{2}$, Kimie Fujita ${ }^{3}$, Akihiko Miyatake $^{4}$, \\ Satoru Doi ${ }^{5}$, Yoichi Kohno ${ }^{2}$, Yoshitaka Okamoto ${ }^{6}$, Tomomitsu Hirota ${ }^{7}$, Mayumi Tamari ${ }^{7}$, Akira Hata ${ }^{1}$ \\ and Yoichi Suzuki ${ }^{1}$
}

In asthma genetics, the association of highly replicated susceptibility genes lacks consistency across populations. To identify genuine associations, we investigated the reproducibility of the 23 most promising asthma and asthma-related candidate genes in a moderately sized sample from the Japanese population. We compared the frequency of 33 polymorphisms in unrelated cases and controls and tested for their association with asthma, atopy and serum total IgE levels using allele frequency, codominant, dominant and recessive genotype models. On the basis of the consistency of our findings with previous metaanalyses and large replication studies, IL13, TNF, ADAM33, IL4RA and TBXA2R might represent common major asthma and asthma-related trait genes. Individual gene assessment was extended to the interactions between two polymorphisms using our original method. Interactions between TBXA2R and ADAM33, and IL4RA and C3 were suggested to increase the risk for childhood and all asthma (adult and childhood asthma combined). The confirmation of previously reported associations between gene polymorphisms and phenotypes was problematic when as few as several hundred samples per group were used. Stratification of the subjects by environmental factors or other confounding factors may be necessary to improve the sensitivity and reliability of association results.

Journal of Human Genetics (2010) 55, 342-349; doi:10.1038/jhg.2010.32; published online 16 April 2010

Keywords: association; asthma; atopy; polymorphism; replication

\section{INTRODUCTION}

Asthma is a heritable trait ${ }^{1}$ and investigations to determine the genetic components underlying asthma using linkage mapping and the candidate gene approach have been carried out. By 2006, more than 100 genes were associated with asthma and asthma-related phenotypes; ${ }^{2} 25$ of these genes have been replicated in more than six independent association studies. In 2008, this list was complemented with an additional three genes, FLG, NAT2 and CCL15. ${ }^{3}$ However, no single polymorphic marker or gene locus has been unanimously labeled as a strong and independent genetic determinant of asthma, and the results for the highly replicated genes have been inconsistent across the tested populations. ${ }^{3}$

To identify true associations, it is of critical importance to comprehensively replicate the initial finding. ${ }^{4}$ To this aim, we investigated whether the 23 most replicated genes for asthma and asthma-related phenotypes were positively associated with extrinsic childhood and adult asthma, atopy and total serum IgE levels in a moderately sized sample drawn from the Japanese population.

We also tested eight genes that were significantly associated with asthma in our subjects: IL $13,{ }^{5}$ TBXA2R, ${ }^{6}$ GSTP $1,{ }^{7}$ ADAM $33,{ }^{8}$ MMP,${ }^{9}$ IL12B, ${ }^{10} C 3^{11}$ and SOCS1. ${ }^{12}$ The re-evaluation of these associations is conditioned by the limitations of the original reports in which childhood asthma included subjects with nonatopic asthma and those who were $<4$ years of age. The adult asthma cases also included nonatopic asthma in some of these reports. Moreover, the comparison of the childhood asthma group was with an adults-only control group. In this study, we redefined the atopic asthma patients, introduced agematched child controls and re-evaluated the association of these genes with the asthma phenotypes, atopy and total serum IgE levels.

Further, we extended the assessment of individual genes to identify potential interactions between the genes, as increasing knowledge about biological pathways and gene networks implies that genegene interactions are important and must be taken into account when estimating the genetic risk of a disease. ${ }^{13}$

\section{MATERIALS AND METHODS}

Study population

The asthma population was restricted to extrinsic asthma patients with subsequent distinction between childhood and adult asthma by cutoff age of below or above 16 years old regardless of the age of the disease onset. We recruited 325 subjects with childhood atopic asthma, 367 adults with atopic

${ }^{1}$ Department of Public Health, Graduate School of Medicine, Chiba University, Chiba, Japan; ${ }^{2}$ Department of Pediatrics, Graduate School of Medicine, Chiba University, Chiba, Japan; ${ }^{3}$ School of Human Nursing, The University of Shiga Prefecture, Shiga, Japan; ${ }^{4}$ Miyatake Asthma Clinic, Osaka, Japan; ${ }^{5}$ Department of Pediatric Allergy, Osaka Prefectural Medical Center for Respiratory and Allergic Diseases, Osaka, Japan; ${ }^{6}$ Department of Otolaryngology, Graduate School of Medicine, Chiba University, Chiba, Japan and ${ }^{7}$ Laboratory for Respiratory Diseases, Center for Genomic Medicine, RIKEN, Kanagawa, Japan Correspondence: Dr Y Suzuki, Department of Public Health, Chiba University Graduate School of Medicine, 1-8-1 Inohana, Chuo-ku, Chiba 260-8670, Japan. E-mail: ysuzuki@faculty.chiba-u.jp

Received 30 November 2009; revised 15 March 2010; accepted 24 March 2010; published online 16 April 2010 
asthma and 646 adult controls from Osaka City, Japan. The details of these subjects have been described elsewhere. ${ }^{10,12,14}$ For childhood controls and the investigation of total and specific IgE levels, we recruited children attending an elementary school in Chiba City, Japan. The clinical characteristics of this population as well as inclusion and exclusion criteria have been described previously. ${ }^{15}$ In brief, after the exclusion of questionnaire-assessed asthma and/ or atopic dermatitis subjects and those with congenital heart diseases and lung diseases caused by premature birth, 336 children having a complete set of information on the total and eight specific IgE levels, genotypes and environmental factors were assigned to the child control group. The mean ages (range) of the four groups were as follows: childhood asthma, 9.9 (4-15); adult asthma, 45 (16-83); child controls, 9.3 (6-12); and adult control, 43.7 (20-75) years. Written informed consent was received from all participants and the study was approved by the ethics committees of Chiba University Graduate School of Medicine and RIKEN.

\section{Gene and polymorphism selection}

The list of candidate gene polymorphisms included in this study, their location within the gene and corresponding rs numbers are given in Supplementary Table S1. In this table, we also included the allele frequency in child and adult control populations.

The most replicated genes were selected based on the list created by Ober and Hoffjan. ${ }^{2}$ From the 25 cited genes, we intentionally excluded HLA-DRB1 and $H L A-D B P 1$ from our analysis due to the high number of variants linked to asthma and asthma-related phenotypes, the genotyping of which would surpass our capacity. The remaining 23 genes were tested for association based on the most positively reported polymorphisms and are represented by Group 1 (>10) and Group 2 (6-10), depending on the number of their replications (Supplementary Table S1). We included four genes (MMP9, IL12B, C3 and SOCS1), which were not in the original 23 genes, but were found to be associated with asthma in our previous studies (Supplementary Table S1, Group 3). These were tested for association with the same positive polymorphisms as in the initial reports.

\section{DNA extraction and genotyping}

Genomic DNA was prepared from peripheral blood samples using the standard protocols. Whole genome amplification was carried out using the Illustra GenomiPhi V2 DNA Amplification Kit (GE Healthcare, Buckinghamshire, UK) according to the manufacturer's instructions. Genotyping was carried out by means of allele-specific amplification, single-nucleotide primer extension reaction or fragment analysis of the PCR product. The genotyping methods used for each polymorphism are given in Supplementary Table S1 and the primer sequences are shown in Supplementary Tables S2, S3a, S3b and S4. The results obtained by these molecular assays were analyzed on ABI PRISM 3100 Genetic Analyzer and ABI PRISM 7000 Sequence Detector Systems (Applied Biosystems, Foster City, CA, USA), or by using Chromo4 Real-Times System (BioRad Laboratories, Hercules, CA, USA). The detailed genotypic protocols are described in Supplementary Methods.

\section{Statistical analysis}

A case-control study design was used to analyze the associations between gene variants and the dichotomous phenotypes. We calculated allele frequencies and tested their agreement with Hardy-Weinberg equilibrium (HWE) using a $\chi^{2}$ goodness-of-fit test. We compared differences in the allele frequencies and genotype distribution of each polymorphism between the case and control subjects by using a $2 \times 2$ (allele) or $2 \times 3$ contingency $\chi^{2}$-test (dominant, codominant and recessive genotype models) with 1 or 2 degree of freedom. Total serum IgE values were logarithm transformed to approximate a normal distribution and analyzed as a quantitative trait using two different tests: linear regression analysis with age and sex as covariates; and analysis of variance (ANOVA). All statistical analyses were carried out with SPSS Statistics 17.0 (SPSS Japan Corporation, Tokyo, Japan). The statistical power of this study was calculated using SamplePower 2.0 (SPSS Corporation). $P$-values $<0.05$ were judged to be significant; as there was an a priori hypothesis with all tested polymorphisms, we did not adjust the significance levels for multiple testing.
Interactions between two polymorphisms were screened using a program written in Excel VBA (Microsoft Japan, Tokyo, Japan). This program identified every possible pairing of polymorphisms, calculated the $\chi^{2}$ and $P$-values of contingency tables between the genotype of one polymorphism and the disease after stratification by the genotype of the other polymorphism. The program then calculated the $\chi^{2}$ and $P$-values for the goodness-of-fit statistics of the distribution pattern of genotype vs disease table with stratification against no stratification. Single-nucleotide polymorphisms (SNPs) in the same gene were not tested for this interaction because most of them showed linkage disequilibrium.

\section{RESULTS}

\section{Statistical power of this study}

We estimated the frequency difference between the case and control groups, and odds ratio (OR) for our samples with different genotype/ allele frequencies according to sample size. We set our detection power at $80 \%$ with an $\alpha$ level of 0.05 .

Supplementary Table S5a shows the results of this calculation for the asthma cases, when estimation was carried out for given genotype/ allele frequencies among their respective control peers. Adult asthma group had slightly better power than the childhood asthma group because of almost twice bigger number of adult controls compared with the child controls. In the case of adult asthma, enough $(>80 \%)$ power could be expected for polymorphisms with an OR of 1.4 when the minor genotype frequency was from $30-60 \%$. In the allele frequency comparison, there was slightly more detection power than for the genotype comparison, as the number of alleles was twice that of the genotype. When we estimated the genotype/allele frequencies in child asthmatics for given adult control genotype/allele frequencies (Supplementary Table S5b), as expected, some increase in power could be observed with ORs getting closer to those of childhood asthma vs child control estimation. In case of calculation based on the comparison between combined asthma subjects and combined controls (Supplementary Table S5b), ORs of 1.3 and 1.2 could be detected for minor genotype and allele frequencies of 30-60\%, respectively.

The estimated power to detect an association between atopy and genetic polymorphisms (Supplementary Table S6) was comparatively inferior to that of asthma with an OR of 1.9.

Association of the $\mathbf{2 3}$ genes with childhood and adult atopic asthma The genotype frequencies of all gene variants, except for GSTM1 ins/ del, were in Hardy-Weinberg equilibrium (goodness-of-fit $\chi^{2}$-test, $P>0.01$ ) in adult and child controls. We could not test the GSTM1 ins/del variant for Hardy-Weinberg equilibrium because the genotyping method did not distinguish between ins/del heterozygotes and homozygotes.

The summary of results for basic comparison between cases and controls is presented in Table 1 , in which only polymorphisms with $P$ value $<0.05$ in at least one genetic model test are shown. When the frequency of polymorphisms in childhood asthmatics were compared with those of asthma free children, the most strong candidate variant was IL13-1112C > T, which was significantly associated in all but dominant genotype model; LTC4S was positively associated in the allele frequency and dominant model tests and CCL5 $-28 \mathrm{C}>\mathrm{G}$ resulted in significant difference only in the dominant genotype model. In the adult samples, there were significant differences noted between the controls and the asthma patients for TNF, ADAM33 and NOS1. TNF showed positive results in the allele frequency, codominant and dominant models. Association of ADAM33 Met764Thr variant with adult asthma was significant for allele frequency and dominant genotype models. There was strong association of both NOS1 variants with the disease in all tests except for GT repeat intron 
Table 1 Association of genotyped polymorphisms with the asthma phenotypes (basic comparisons)

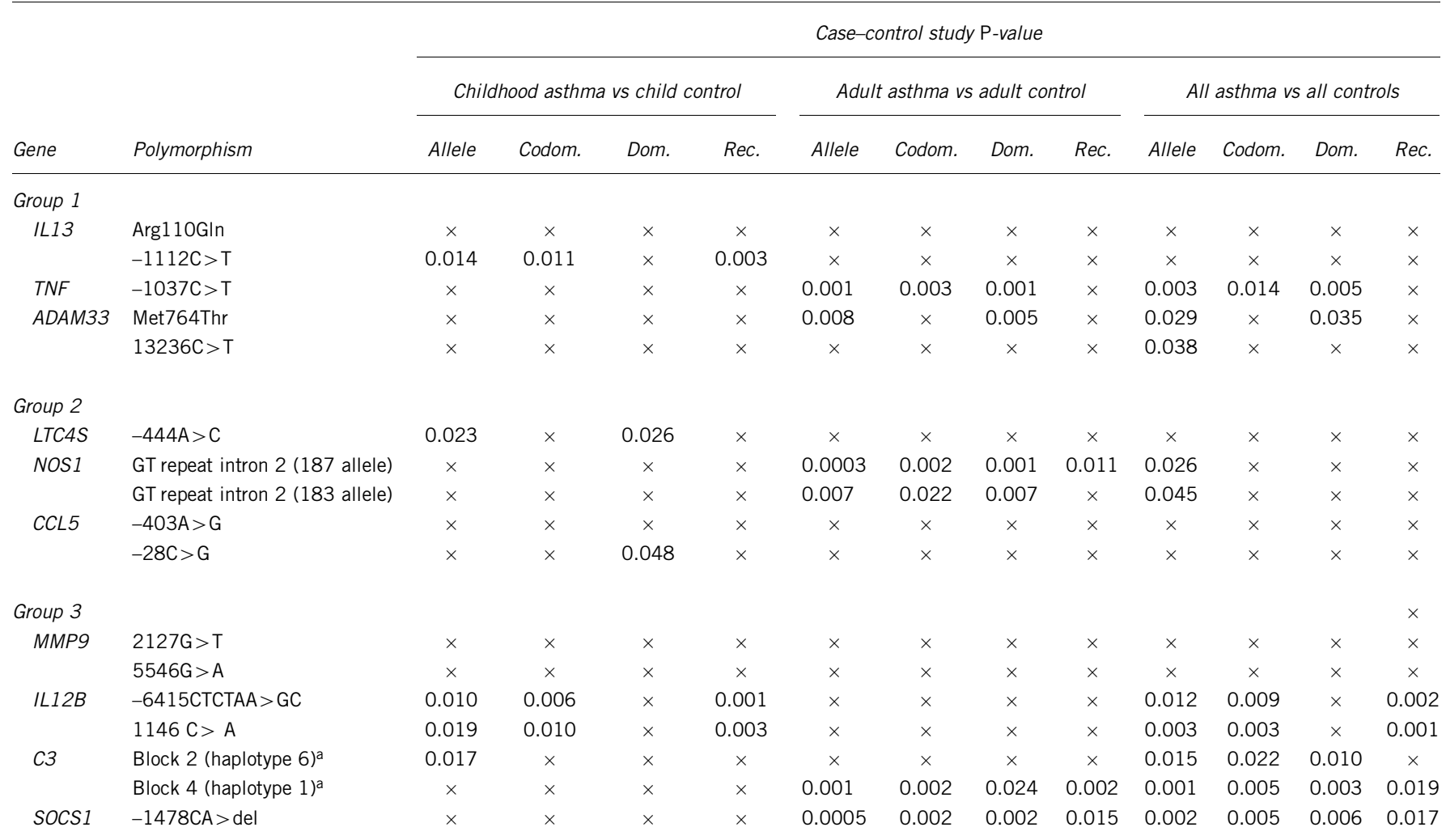

Abbreviations: Allele, $\chi^{2}$-test of allele frequency; codom., $2 \times 3$ codominant model genotype $\chi^{2}$-test; dom., $2 \times 2$ dominant model genotype $\chi^{2}$-test; rec., $2 \times 2$ recessive model genotype $\chi^{2}$-test. aFor haplotype description please refer Inoue et al. ${ }^{11}$

Polymorphisms with a $P$-value $\geqslant 0.05$ in all association tests of the four genetic models are not shown.
$\times P \geqslant 0.05$.

(183 allele) in the recessive genotype model. In the association test between the polymorphisms and all asthma, that is child and adult asthma combined, significant associations were observed for the same genes as in adult asthma. TNF and ADAM33 Met764Thr remained positively associated with asthma in the allele frequency, codominant and dominant models alike in the single adult asthma group comparison. The association of NOS1 variants became weaker with significance only in allele frequency model. Additional significant association was observed only for ADAM33 $13236 \mathrm{C}>\mathrm{T}$ in the allele frequency model.

Conditioned by the inherent characteristics of our case and control samples, we extended our analysis further (Table 2). In our child control group, we recognize the potential presence of asthma susceptibility gene variants carriers who might become asthmatics later in life, and consequently, to become a source of bias. Addressing this issue, we tested childhood asthmatics also with adult controls, considering the later ones as more reliable asthma 'free' subjects. Paradoxically, the previous significant associations found in the comparison for child asthma vs child control disappeared; instead, TNF, IL4RA, ADAM33, GSTM1, AAA1 and NOS1 showed significant results. The substantial difference in results between childhood asthma vs child control and childhood asthma vs adult control tests might be due to some confounding effects of hidden genetic and environmental heterogeneities between child and adult control groups.

Furthermore, as the natural history of asthma in some adults starts during childhood, we characterized the association results after stratifying adult asthma cases by age at the onset (Table 2). Using the same cutoff age ( $<16$ years) as for discerning childhood asthma from adult asthma, we obtained 118 adults (32\% of adult asthma), who have reported physician diagnosed asthma during their childhood, and 249 adults with newly onset bronchial asthma. To elucidate the genes associated with differential onset of asthma in children, we merged the child-onset adult subgroup with childhood asthma group (all L16 adult atopic asthma (BA)) and tested with either child or adult controls by applying the same strategy as used in the nonstratified adult asthma. In the association test with child control, besides the previously detected gene variants in the comparison of child asthma vs child control, TNF showed at least one significant association in four association tests, whereas the significance of CCL5 disappeared. When the combined child-onset asthma group was compared with adult controls, the observed associations in child asthma vs adult control comparison could be confirmed for only IL4RA Ile50Val and ADAM33 13236C $>\mathrm{T}$ polymorphisms. We next examined the new-onset adult asthma, in which TNF and ADAM33 Met764Thr have retained their significant associations found with the onset nonstratified adult asthma. In addition, IL13, IL4RA and GSTM1 have emerged as genes associated with asthma establishment during adulthood. Interestingly, the two ADAM33 polymorphisms were associated with different asthma phenotypes when comparisons were carried out with adult control. ADAM33 Met764Thr was significantly associated with adult asthma, whereas ADAM33 $13236 \mathrm{C}>\mathrm{T}$ variant was significantly more frequent in childhood asthma and all child-onset asthma groups.

We also compared the distribution of polymorphisms between the two control groups; it showed a relative homogeneity with significant 
Table 2 Summary of the association results between polymorphisms and asthma when case and control groups were compared in various combinations

\begin{tabular}{|c|c|c|c|c|c|c|c|c|c|}
\hline \multirow[b]{2}{*}{ Gene } & \multirow[b]{2}{*}{ Polymorphism } & \multicolumn{8}{|c|}{ Two group comparison } \\
\hline & & $\begin{array}{c}\text { Child BA vs } \\
\text { child CO }\end{array}$ & $\begin{array}{c}\text { Child BA vs } \\
\text { adult } C O\end{array}$ & $\begin{array}{c}\text { Adult } B A \text { vs } \\
\text { adult } C O\end{array}$ & $\begin{array}{c}\text { All } L 16 B A \text { vs } \\
\text { child CO }\end{array}$ & $\begin{array}{c}\text { Al/ } L 16 B A \text { vs } \\
\text { adult CO }\end{array}$ & $\begin{array}{c}\text { M16 adult BA } \\
\text { vs adult CO }\end{array}$ & $\begin{array}{c}\text { Child CO vs } \\
\text { adult } \mathrm{CO}\end{array}$ & $\begin{array}{c}\text { Child } B A \text { vs } \\
\text { adult } B A\end{array}$ \\
\hline \multicolumn{10}{|l|}{ Group 1} \\
\hline \multirow[t]{2}{*}{ IL13 } & Arg110GIn & $\times$ & $x$ & $\times$ & $\times$ & $\times$ & $x$ & $\times$ & $\times$ \\
\hline & $-1112 \mathrm{C}>\mathrm{T}$ & 0 & $x$ & $\times$ & 0 & $x$ & 0 & 0 & $\times$ \\
\hline$T N F$ & $-1037 \mathrm{C}>\mathrm{T}$ & $\times$ & 0 & 0 & 0 & $\times$ & 0 & $\times$ & $\times$ \\
\hline \multirow[t]{2}{*}{ ILARA } & Ile50Val & $\times$ & 0 & $\times$ & $\times$ & 0 & 0 & $\times$ & $\times$ \\
\hline & Gln551Arg & $x$ & $x$ & $\times$ & $\times$ & $x$ & $x$ & $\times$ & 0 \\
\hline \multirow[t]{2}{*}{ ADAM33 } & Met764Thr & $\times$ & $x$ & 0 & $x$ & $\times$ & 0 & $\times$ & 0 \\
\hline & $13236 \mathrm{C}>\mathrm{T}$ & $\times$ & 0 & $\times$ & $\times$ & 0 & $\times$ & $\times$ & 0 \\
\hline \multicolumn{10}{|l|}{ Group 2} \\
\hline GSTM1 & Ins/del & $\times$ & 0 & $\times$ & $\times$ & $\times$ & 0 & $\times$ & 0 \\
\hline IL10 & $571 C>A$ & $\times$ & $\times$ & $\times$ & $\times$ & $\times$ & $\times$ & $\times$ & 0 \\
\hline LTC4S & $-444 A>C$ & 0 & $\times$ & $x$ & 0 & $\times$ & $x$ & 0 & $\times$ \\
\hline$A A A 1$ & $522363 G>C$ & $\times$ & 0 & $\times$ & $\times$ & $\times$ & $\times$ & $\times$ & 0 \\
\hline \multirow[t]{2}{*}{ NOS1 } & GT repeat (187 allele) & $x$ & 0 & 0 & $\times$ & $x$ & $x$ & $\times$ & 0 \\
\hline & GT repeat (183 allele) & $\times$ & $\times$ & 0 & $\times$ & $x$ & $\times$ & $\times$ & $\times$ \\
\hline CCL5 & $-28 C>G$ & 0 & $\times$ & $\times$ & $\times$ & $\times$ & $\times$ & $\times$ & $\times$ \\
\hline \multicolumn{10}{|l|}{ Group 3} \\
\hline \multirow[t]{2}{*}{ MMP9 } & $2127 \mathrm{G}>\mathrm{T}$ & $\times$ & o & $\times$ & $\times$ & $\times$ & 0 & 0 & $\times$ \\
\hline & $5546 \mathrm{G}>\mathrm{A}$ & $\times$ & 0 & $\times$ & $\times$ & 0 & $\times$ & 0 & $\times$ \\
\hline \multirow[t]{2}{*}{$I L 12 B$} & $-6415 \mathrm{ins} / \mathrm{del}$ & 0 & o & $x$ & 0 & 0 & $x$ & $\times$ & 0 \\
\hline & $1146 C>A$ & 0 & o & $\times$ & 0 & 0 & $\times$ & $\times$ & 0 \\
\hline \multirow[t]{2}{*}{ C3 } & Block 2 (haplotype 6) & 0 & $\times$ & $\times$ & 0 & 0 & $\times$ & $\times$ & $\times$ \\
\hline & 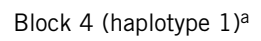 & $\times$ & 0 & 0 & $\times$ & o & $x$ & $\times$ & $x$ \\
\hline SOCS1 & $-1478 \mathrm{CA}>$ del & $\times$ & $\times$ & 0 & $\times$ & 0 & $\times$ & $\times$ & $\times$ \\
\hline
\end{tabular}

Abbreviations: Child BA, childhood atopic asthma; child CO, child controls; adult BA, adult atopic asthma; adult CO, adult controls; all L16 adult BA, childhood atopic asthma and adult atopic asthma at onset age $<16$ years combined; M16 adult BA, adult atopic asthma at onset age $\geqslant 16$. ${ }^{a}$ For haplotype description please refer Inoue et al. ${ }^{11}$

Polymorphisms with a $P$-value $\geqslant 0.05$ in all association tests of the four genetic models are not shown.

${ }^{\circ} P$-value $<0.05$ in at least one association test of the four genetic models.

${ }^{\times}$None of the $P$-value in the four genetic models was $<0.05$.

difference only for IL13 and LTC4S. It indicates that there would be little bias created using either sample as a control group for any of the case groups and justifies our combining of the two control groups to form a single one in the comparison for all asthma vs all control. In the comparison test for childhood asthma vs adult asthma, IL4RA, ADAM33, GSTM1, IL10, AAA1 and NOS1 genes were found significantly different, which is coherent with the case-control comparison results.

\section{Association of the $\mathbf{2 3}$ genes with atopy}

ADAM33 13236C $>$ T, NOS1 GT repeat 183 bp allele and TBXA2R $795 \mathrm{~T}>\mathrm{C}$ were significantly associated with atopy in the recessive or dominant models (Supplementary Table S7). NOS1 and TBXA2R also showed significant association in the allele frequency test, whereas ADAM33 did not.

\section{Association of the 23 genes with total serum IgE}

Supplementary Table S8 summarizes the relationship between the mean of $\log _{10}$-transformed total serum IgE level and gene variants. There was a significant association between GSTP1 and total IgE level as determined by ANOVA that remained significant after adjusting for age and gender as covariates in linear regression analysis.
Re-evaluation of the associations previously discovered in our subjects

We confirmed the association of the IL13, ADAM33, MMP9, IL12B, C3 and SOCS1 polymorphisms with atopic asthma (Tables 1 and 2). The association of $T B X A 2 R$ with asthma was not replicated; instead, we identified an association of TBXA2R with atopy (Supplementary Table S7). Comparatively to our previous studies, in this study we also investigated the polymorphisms for association with serum IgE level. Significant results were observed for GSTP1 as described above and for MMP9 5546G >A (Supplementary Table S8).

\section{Screening of gene-gene interactions}

From the 40 genetic polymorphisms, polymorphism pairs with a $P$-value for association with the disease of $<0.05$ and a $P$-value for interaction $<0.01$ are listed in Table 3. A pair of LTA and TNF SNPs showed a very small $P$-value; however, these two genes are physically close and these SNPs are known to be in linkage disequilibrium. Thus, this combination was omitted from the table. Interactions between TBXA2R and ADAM33 and between IL4RA and C3 were suggested for childhood asthma and all asthma. These interactions were our most robust results. 


\begin{tabular}{|c|c|c|c|c|c|c|c|c|}
\hline \multirow[b]{2}{*}{ SNP 1} & \multirow[b]{2}{*}{ SNP 1 genotype } & \multirow[b]{2}{*}{ SNP 2} & \multicolumn{3}{|c|}{$\begin{array}{c}\text { Effect of SNP } 1 \text { on association } \\
\text { between SNP } 2 \text { and disease } e^{\mathrm{a}}\end{array}$} & \multicolumn{3}{|c|}{$\begin{array}{l}\text { Association between SNP } 2 \text { and } \\
\text { disease after stratification by SNP } 1\end{array}$} \\
\hline & & & $\chi^{2}$ & d.f. & P-value & $\chi^{2}$ & d.f. & P-value \\
\hline \multicolumn{9}{|l|}{ Childhood asthma } \\
\hline LTA $-735 \mathrm{G}>\mathrm{A}$ & $A / A$ & $I L 12 B 1146 C>A$ & 7.12 & 1 & 0.0076 & 18.1 & 2 & 0.00012 \\
\hline IL4RA Ile50Val & Ile/lle & C3 block2 (haplotype 6) & 11.63 & 1 & 0.00064 & 13.42 & 1 & 0.00025 \\
\hline$A A A 1522363 \mathrm{G}>C$ & $\mathrm{C} / \mathrm{C}$ & $I L 433 C>T$ & 10.98 & 2 & 0.0041 & 8.88 & 2 & 0.012 \\
\hline$A A A 1522363 G>C$ & $\mathrm{C} / \mathrm{C}$ & IL4 -590T>C & 9.31 & 2 & 0.0095 & 7.67 & 2 & 0.022 \\
\hline$C C L 5-28 C>G$ & $G / G$ & IL13 Arg110GIn & 7.2 & 1 & 0.0073 & 4.69 & 1 & 0.03 \\
\hline$C D 14-550 C>T$ & $T / T$ & IL $10-571 \mathrm{C}>\mathrm{A}$ & 7.1 & 1 & 0.0077 & 4.11 & 1 & 0.043 \\
\hline \multicolumn{9}{|l|}{ Adult asthma } \\
\hline \multicolumn{9}{|l|}{ All asthma } \\
\hline TBXA2R 924C > T & $\mathrm{C} / \mathrm{T}$ & ADAM33 313236C $>\mathrm{T}$ & 12.76 & 2 & 0.0017 & 11.83 & 2 & 0.0027 \\
\hline IL4RA Ile50Val & Ile/lle & C3 block2 (haplotype 6) & 7.29 & 1 & 0.0069 & 6.62 & 1 & 0.01 \\
\hline ADAM33 $313236 \mathrm{C}>\mathrm{T}$ & $\mathrm{C} / \mathrm{T}$ & $T B X A 2 R$ 924C $>\mathrm{T}$ & 8.05 & 1 & 0.0046 & 8.99 & 2 & 0.011 \\
\hline$T N F-1037 C>T$ & $\mathrm{~T} / \mathrm{T}$ & $C D 14-159 C>T$ & 7.04 & 1 & 0.008 & 7.34 & 2 & 0.026 \\
\hline STAT6 GT repeat & $172 / 172$ & $\operatorname{CC16} 38 \mathrm{~A}>\mathrm{G}$ & 11.01 & 2 & 0.0041 & 6.9 & 2 & 0.036 \\
\hline
\end{tabular}

Abbreviations: d.f., degree of freedom; SNP, single-nucleotide polymorphism.

apolymorphism combination that demonstrates the interactions with $P<0.01$ and an association with $P<0.05$ are shown.

bFor haplotype description please refer Inoue et al. ${ }^{11}$

\section{DISCUSSION}

We conducted our study in three phases. We first, under the assumption of the common disease-common variant hypothesis, investigated whether the 23 most promising asthma/atopy candidate genes retained their association in a Japanese population set. We considered the gene as the unit of our replication, and the gene was judged as positively replicated if demonstrated a statistically significant association with one or more phenotypes (atopic asthma, atopy and total serum IgE level) in at least one of four genetic models. In our samples, among the highly replicated genes ( $>10$ positive associations), IL13, TNF, IL4RA and ADAM33 maintained their reputation as robust asthma and asthma-related candidate genes. From the genes with a lower replication rate (6-10), we confirmed the associations of GSTM1, GSTP1, LTC4S, AAA1, NOS1, CCL5 and TBXA2R. In the second phase of the study, we screened our initial significant asthma associations to IL13, TBXA2R, GSTP1, ADAM33, MMP9, IL12B, C3 and SOCS1. These associations were replicated for all genes, except for $T B X A 2 R$ and GSTP1, which were associated with atopy and total serum IgE level, respectively. In the final phase, we explored the potential multigenic effect of all 27 candidate genes (the three groups of genes combined) in the expression of asthma phenotypes based on a pairwise method.

If we summarize our findings from the replication study, interesting patterns of associations could be observed. Except for IL13 and IL4RA, we found no association for the prominent genes implicated in innate immunity and immunoregulation with asthma or asthma-related phenotypes, that is, CD14, IL10 and TGFB1 as well as the Th2 cytokines and their receptors represented by IL4, STAT6 and MS4A2. Instead, the genes secreted from airway epithelial cells (for example, CCL5 and $A A A 1$ ), and genes known to affect lung function, mediate inflammatory conditions and participate in airway remodeling (for example, TNF, ADAM33, GSTM1, GSTP1, LTC4S, NOS1 and $T B X A 2 R)$ demonstrated statistically significant associations. This observation may indicate the relatively higher predisposing effect that these two groups of genes exert on the development of asthmarelated phenotypes in the Japanese population. Our postulation is supported by the hypothesis that not the dysregulated immune response, but the inherently abnormal respiratory epithelium of asthmatics and the reactivation of the epithelial-mesenchymal trophic unit leading to pathological airway wall remodeling has a major role in the disease. ${ }^{16,17}$ Another interesting finding was the distinct partition of genes between adult and child asthma. The association of LTC4S, $A A A 1$ and CCL5 specifically in the child samples might reflect the differing etiopathogenetic background of childhood asthma. ${ }^{18,19}$ For example, CCL5 is a key chemokine recruiting Th1 and Th2 proinflammatory cells, and its expression in epithelial cells is induced by the respiratory syncytial virus (RSV). ${ }^{20,21}$ This is in line with the evidences that the epithelial barrier in young asthmatics is inherently abnormal ${ }^{22}$ and that RSV bronchiolitis is a more important risk factor for the development of asthma and atopy up to the age of 7 years than heredity or environmental factors. ${ }^{23}$ Moreover, the candidate genes showing significant association with both the phenotypes, when the case groups were compared with adult controls separately (childhood asthma vs adult control and adult asthma vs adult control), were the same as those observed in the combined analysis (all asthma vs all controls). Thus, TNF, ADAM33 and NOS1 might represent the common susceptibility gene for adult and childhood asthma. When we categorized asthma cases by the age at onset, the number of shared 
significant genes increased (IL13, TNF, IL4RA and ADAM33), making in the overall no substantial differences in the genetic determinants between child-onset and adult-onset asthmas. The exception was for LTC4S and GSTM1, suggesting the former gene to be related with the development of asthma during childhood and the later one during adulthood. However, as GSTM1 was significantly associated with childhood asthma in the comparison of childhood asthma vs adult control, it is difficult to conclude whether the polymorphism in this gene affected the susceptibility to new-onset adult asthma.

Our replication results were in agreement with several large-scale studies. A recent review of the literature revealed that five asthma candidate genes, ADAM33, TNF, TBXA2R, CD14 and LTC4S, were the focus of several meta-analyses in which ADAM33 and TNF had a modest association with asthma. ${ }^{24}$ The first genome-wide replication study of 39 asthma candidate genes generated IL4RA results that were consistent with our observations. ${ }^{25}$ In the most comprehensive replication study carried out to date, the reproducibility of 93 genes previously associated with asthma and/or asthma intermediate traits was tested. ${ }^{26}$ IL13 was associated with asthma, and TBXA2R was associated with atopy, as we also observed in this study. Our replication rate of $48 \%$ ( 11 genes out of 23 ; OR 1.15-1.62, if the outlier OR of 3.01 (95\% confidence interval, $1.40-6.51$ ) for $I L 13-1112 \mathrm{C}>\mathrm{T}$ is excluded) was higher than that reported in previous large association studies (for OR see Table 4); the study of Daley et al. ${ }^{26}$ (unrelated case-control sample of $N=5565$ ) and a genome-wide screen of 422 nuclear families using SNP arrays had low replication rates of $13 \%$ (12 out of 93 tested genes, OR <1.4) and 15.4\% ( 6 out of 39 at SNP-level replication, OR 1.4-1.7), respectively. This better replication rate might be attributed to our sample size, as it is well documented that smaller studies have a tendency to have more favorable outcomes than larger ones. ${ }^{27}$ Daley et al. concluded that many published associations for asthma and atopy may be false-positive results. Whereas Rogers et al. ${ }^{25}$ suggested that the poor coverage of genome-wide association study genotyping platforms and lack of statistical power due to insufficient sample size were the main reasons for their low replication. We are more inclined to suspect the 'contextual' bias explaining our failure to replicate all candidate genes. By that we mean the confounding effect of the whole complex network of genegene and gene-environmental interactions. This can be seen from the controversy in the findings between this current study and our previous one. In this study, CD14 $-550 \mathrm{C}>\mathrm{T}$ and IL4RA Ile50Val were not associated with total serum IgE level. Whereas, in our recent association study carried out on the same school children, these two gene variants had a modifying effect on the levels of total IgE later in life depending on the children's attendance of day care before 2 years of age. ${ }^{15}$ This association could be detected because the day care attendance was taken in consideration as an environmental factor and the effect of a gene was investigated simultaneously with the effect of the other one.

In the gene-gene interaction analysis, we identified some statistical interactions that asserted the weak associations found in the individual gene assessment. Among them, significant interaction between $C 3$ and IL4RA and between ADAM33 and TBXA2R were observed for both childhood and all asthma groups. Although straightforward functional evidences of such paired interactions are lacking, some plausibility can be inferred. C3 or complement component 3 is an important part of the innate immunity recognizing exogenous and endogenous molecular patterns. Some functions of its C3a subtype indicate a possible role for the complement system in asthma pathogenesis. ${ }^{28}$ In allergen-sensitized mouse model of pulmonary allergy deficient in $C 3$ or in its receptor $C 3 a R$, Drouin et al. ${ }^{29,30}$ have observed that in the mutant mice the characteristic manifestations of asthma were significantly attenuated compared with wild-type animals and that in the lung the number of interleukin 4 (IL4)producing cells was decreased; whereas Kawamoto et al. ${ }^{31}$ showed that the absence of $C 3 a R$ in mice results in significantly increased level of Th2 cytokines (IL4, IL5 and IL10). In spite of the fact that the two groups' results are contradictory calling for further examination, the observed functional relationship clearly indicates a modulator role of C3 on IL4 cytokine expression. IL4 signal transduction is mediated through the $\alpha$ subunit of the IL4 receptor (IL4RA), which is IL4 specific. Thus, the C3-IL4-IL4RA axis might be one of the plausible models for the interaction between $C 3$ and IL4RA. With regard to $A D A M 33$ and TBXA2R, one common feature that could indicate their putative interaction is their involvement in angiogenesis, a process frequently underestimated in the pathophysiology of asthma. ${ }^{32}$ Novel findings on ADAM33 showed that its catalytic domain promoted endothelial cell (EC) proliferation in vitro, and formation of new vessels ex vivo and in vivo. ${ }^{33}$ TBXA2R is also known to be implicated in neovascularization but in an opposed way: suppresses EC migration and angiogenesis by inhibiting the effector pathways of the vascular endothelial growth factor (VEGF), a key angiogenic and chemotactic regulator of EC. ${ }^{34}$ Although the exact mechanism by which ADAM33 exerts its proangiogenic effect is yet to be elucidated, the involvement of VEGF is likely to take place. In that case, the above findings will suggest interactive effect of ADAM33 and TBXAR on VEGF regulation and consequently on angiogenesis and microvascular remodeling of conductive airways in asthma.

Nevertheless, the significant results of our replication study as well as of the gene-gene interactions investigation should be interpreted with caution for inflation of type 1 errors. We have presented our findings based on the nominal $\alpha$ threshold of $<0.05$ without taking into account multiple testing. Relative to the replication study, this study is not an exploratory study aimed to find a 'significant' gene from multiple candidates but rather to test for confirmation of previously well-established hypotheses. Indeed, the genes from Group 1 and Group 2 are the top asthma and allergy related genes, each replicated in at least six or more independent populations, meaning they all have a high previous probability to show true associations even in the case of a relaxed threshold value for significance. However, if we adjusted for multiple comparisons by the Bonferroni method, none of our significant findings would survive this stringent level of correction. It is obvious that the power is enough to detect genetic effect with OR of around 1.4 with the current sample size, but not if we consider multiple testing. The same is for the results obtained from the screening of the interactions between two polymorphisms. If we strictly applied Bonferroni correction, the significant $P$-values would need to be in the order of $9.46 \times 10^{-5}(0.05 / 528)$ because we carried out $33 C^{2}=528$ tests for each phenotype; no $P$-value reached this value. Thus, our findings for the potential gene-gene interactions must be evaluated physiologically or by analyses of other sets of samples to validate these observations.

There are other limitations to this study. We focused on the effect of genetic polymorphisms on dichotomous phenotypes and ignored clinical severity and environmental factors. There was also a delay between the recruitment of child asthma cases and child control samples, which could be a source of bias due to differences in DNA processing as well as in environmental exposure. Although population stratification was not controlled in this study, we consider the confounding effect of this factor to be of a lesser extent in comparison to studies conducted on North American ${ }^{35,36}$ or Western European $^{37,38}$ populations. From the genetic point of view, this 
Table 4 Odds ratio and $95 \% \mathrm{Cl}$ of significant polymorphisms found in the basic association studies

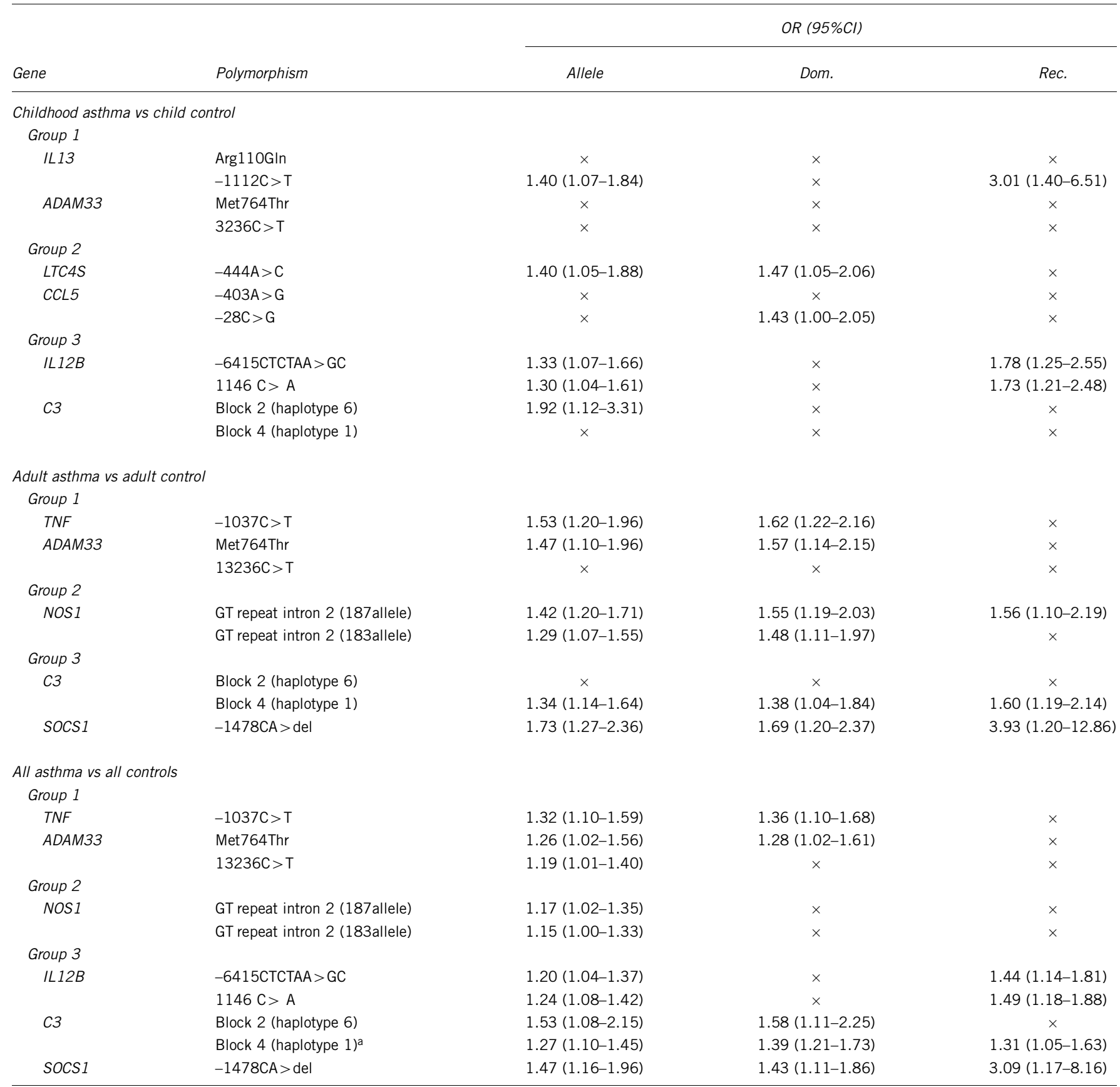

Abbreviations: Allele, $\chi^{2}$-test of allele frequency; $\mathrm{Cl}$, confidence interval; dom., $2 \times 2$ dominant model genotype $\chi^{2}$-test; OR, odds ratio; rec., $2 \times 2$ recessive model genotype $\chi^{2}$-test. aFor haplotype description please refer Inoue et al. ${ }^{11}$

Polymorphisms with a $P$-value $\geqslant 0.05$ in all association tests of the four genetic models are not shown.

Polymorph
$\times P 0.05$

assumption is based on the fact that our control subjects were residents of the mainland of Japan, the population of which belongs to the genetically homogeneous Hondo cluster, ${ }^{39}$ and also on the results of genomic control analysis ${ }^{40}$ that showed the populations from the Kinki and Kanto regions (where we recruited our samples and controls) do not differ in the allele frequency of the null marker. In terms of stratification determined by an individual's socioeconomic position, we would refer to the specific egalitarian characteristic of the Japanese society in support of our claim. ${ }^{41}$
In conclusion, our findings and previous studies suggest that IL13, TNF, IL4RA, ADAM33 and TBXA2R might represent the major asthma and asthma-related traits genes common across populations. GSTM1, GSTP1, LTC4S, AAA1, NOS1 and CCL5 along with MMP9, IL12B, C3 and SOCS1 might be additional susceptibility genes, which have stronger effects in the Japanese population. Despite our failure to replicate the other genes, our results were not strong enough to eliminate them from the candidate gene list because we did not investigate all known variations in these genes and we did not consider 
the effects of environmental factors. Replication studies of genotypephenotype associations with sample sizes ranging from several hundred to several thousand are not exempt from inconsistencies in findings and have low replication rates. Given the present limited availability of biobanks, methodologically irreproachable studies that integrate more detailed clinical information and that explore the effects of genes in their entirety by dissecting the direct and interactive effects from environmental factors and other genes are required to improve the power and reproducibility of genetic association studies.

\section{ACKNOWLEDGEMENTS}

We thank all patients and their families, the volunteers who served as controls and all staff members at the hospitals involved in this study. We also thank Kazuko Hatori, Rieko Yoshida, Yoshiko Hotta and Miyako Takano for their excellent technical assistance. This work was supported by Grants-in-Aid for Scientific Research from the Ministry of Education, Culture, Sports, Science and Technology, Japan, and grants from the Ministry of Health, Labor and Welfare, Japan. We also thank the two referees for their careful reviews, thoughtful comments and their helpful suggestions that greatly improved the paper.

1 Los, H., Koppelman, G. \& Postma, D. The importance of genetic influences in asthma. Eur. Respir. J. 14, 1210-1227 (1999).

2 Ober, C. \& Hoffjan, S. Asthma genetics 2006: the long and winding road to gene discovery. Genes Immun. 7, 95-100 (2006).

3 Vercelli, D. Discovering susceptibility genes for asthma and allergy. Nat. Rev. Immunol. 8, 169-182 (2008).

4 Chanock, S. J., Manolio, T., Boehnke, M., Boerwinkle, E., Hunter, D. J., Thomas, G. et al. Replicating genotype-phenotype associations. Nature 447, 655-660 (2007).

5 Heinzmann, A., Mao, X., Akaiwa, M., Kreomer, R. T., Gao, P., Ohshima, K. et al. Genetic variants of IL-13 signalling and human asthma and atopy. Hum. Mol. Genet. 9, 549559 (2000).

6 Unoki, M., Furuta, S., Onouchi, Y., Watanabe, O., Doi, S., Fujiwara, H. et al. Association studies of 33 single nucleotide polymorphisms (SNPs) in 29 candidate genes for bronchial asthma: positive association a T924C polymorphism in the thromboxane A2 receptor gene. Hum. Genet. 106, 440-446 (2000).

7 Kamada, F., Mashimo, Y., Inoue, H., Shao, C., Hirota, T., Doi, S. et al. The GSTP1 gene is a susceptibility gene for childhood asthma and the GSTM 1 gene is a modifier of the GSTP1 gene. Int. Arch. Allergy Immunol. 144, 275-286 (2007).

8 Hirota, T., Hasegawa, K., Obara, K., Matsuda, A., Akahoshi, M., Nakashima, K. et al. Association between ADAM33 polymorphisms and adult asthma in the Japanese population. Clin. Exp. Allergy 36, 884-891 (2006).

9 Nakashima, K., Hirota, T., Obara, K., Shimizu, M., Doi, S., Fujita, K. et al. A functional polymorphism in MMP-9 is associated with childhood atopic asthma. Biochem. Biophys. Res. Commun. 344, 300-307 (2006)

10 Hirota, T., Suzuki, Y., Hasegawa, K., Obara, K., Matsuda, A., Akahoshi, M. et al. Functional haplotypes of IL-12B are associated with childhood atopic asthma. J. Allergy Clin. Immunol. 116, 789-795 (2005).

11 Inoue, H., Mashimo, Y., Funamizu, M., Shimojo, N., Hasegawa, K., Hirota, T. et al. Association study of the $\mathrm{C} 3$ gene with adult and childhood asthma. J. Hum. Genet. 53, 728-738 (2008).

12 Harada, M., Nakashima, K., Hirota, T., Shimizu, M., Doi, S., Fujita, K. et al. Functional polymorphism in the suppressor of cytokine signaling 1 gene associated with adult asthma. Am. J. Respir. Cell Mol. Biol. 36, 491-496 (2007).

13 Phillips, P. C. Epistasis-the essential role of gene interactions in the structure and evolution of genetic systems. Nat. Rev. Genet. 9, 855-867 (2008).

14 Hasegawa, K., Tamari, M., Shao, C., Shimizu, M., Takahashi, N., Mao, X. Q. et al. Variations in the C3, C3a receptor, and C5 genes affect susceptibility to bronchial asthma. Hum. Genet. 115, 295-301 (2004).

15 Suzuki, Y., Hattori, S., Mashimo, Y., Funamizu, M., Kohno, Y., Okamoto, Y. et al. CD14 and IL4R gene polymorphisms modify the effect of day care attendance on serum IgE levels. J.Allergy Clin. Immunol. 123, 1408-1411.e1 (2009).
16 Holgate, S. T. Epithelium dysfunction in asthma. J. Allergy Clin. Immunol. 120, 1233-1244 (2007).

17 Baldwin, L. \& Roche, W. R. Does remodelling of the airway wall precede asthma? Paediatr. Respir. Rev. 3, 315-320 (2002).

18 Jenkins, H. A., Cherniack, R., Szefler, S. J., Covar, R., Gelfand, E. W. \& Spahn, J. D. A comparison of the clinical characteristics of children and adults with severe asthma. Chest 124, 1318-1324 (2003).

19 Gelfand, E. W. Pediatric asthma: a different disease. Proc. Am. Thorac. Soc. 6, 278-282 (2009).

20 Culley, F. J., Pennycook, A. M. J., Tregoning, J. S., Dodd, J. S., Walzl, G., Wells, T. N. et al. Role of CCL5 (RANTES) in viral lung disease. J. Virol. 80, 8151-8157 (2006).

21 Murai, H., Terada, A., Mizuno, M., Asai, M., Hirabayashi, Y., Shimizu, S. et al. IL-10 and RANTES are elevated in nasopharyngeal secretions of children with respiratory syncytial virus infection. Allergol. Int. 56, 157-163 (2007).

22 Martinez, F.D. The origins of asthma and chronic obstructive pulmonary disease in early life. Proc. Am. Thorac. Soc. 6, 272-277 (2009).

23 Sigurs, N., Bjarnason, R., Sigurbergsson, F. \& Kjellman, B. Respiratory syncytial virus bronchiolitis in infancy is an important risk factor for asthma and allergy at age 7. Am. J. Respir. Crit. Care Med. 161, 1501-1507 (2000).

24 Contopoulos-Ioannidis, D. G., Kouri, I. N. \& Ioannidis, J. P. Genetic predisposition to asthma and atopy. Respiration 74, 8-12 (2007).

25 Rogers, A. J., Raby, B. A., Lasky-Su, J. A., Murphy, A., Lazarus, R., Klanderman, B. J. et al. Assessing the reproducibility of asthma candidate gene associations, using genome-wide data. Am. J. Respir. Crit. Care Med. 179, 1084-1090 (2009).

26 Daley, D., Lemire, M., Akhabir, L., Chan-Yeung, M., He, J. Q., McDonald, T. et al. Analyses of associations with asthma in four asthma population samples from Canada and Australia. Hum. Genet. 125, 445-459 (2009).

27 loannidis, J. P., Trikalinos, T. A., Ntzani, E. E. \& Contopoulos-loannidis, D. G. Genetic associations in large versus small studies: an empirical assessment. Lancet 361, 567-571 (2003).

28 Humbles, A. A., Lu, B., Nilsson, C. A., Lilly, C., Israel, E., Fujiwara, Y. et al. A role for the $\mathrm{C} 3 \mathrm{a}$ anaphylatoxin receptor in the effector phase of asthma. Nature 406, 9981001 (2000).

29 Drouin, S. M., Corry, D. B., Kildsgaard, J. \& Wetsel, R. A. Cutting edge: the absence of C3 demonstrates a role for complement in Th2 effector functions in a murine model of pulmonary allergy. J. Immunol. 167, 4141-4145 (2001).

30 Drouin, S. M., Corry, D. B., Hollman, T. J., Kildsgaard, J. \& Wetsel, R. A. Absence of the complement anaphylatoxin C3a receptor suppresses Th2 effector functions in a murine model of pulmonary allergy. J. Immunol. 169, 5926-5933 (2002).

31 Kawamoto, S., Yalcindag, A., Laouini, D., Brodeur, S., Bryce, P., Lu, B. et al. The anaphylatoxin $\mathrm{C} 3 \mathrm{a}$ downregulates the Th2 response to epicutaneously introduced antigen. J. Clin. Invest. 114, 399-407 (2004).

32 Bischof, R. J., Bourke, J. E., Hirst, S. J., Meeusen, E. N. T., Snibson, K. J. \& Van Der Velden, J. Measurement and impact of remodeling in the lung: airway neovascularization in asthma. Proc. Am. Thorac. Soc. 6, 673-677 (2009).

33 Puxeddu, I., Pang, Y. Y., Harvey, A., Haitchi, H. M., Nicholas, B., Yoshisue, H. et al. The soluble form of a disintegrin and metalloprotease 33 promotes angiogenesis: implications for airway remodeling in asthma. J. Allergy Clin. Immunol. 121, 1400-1406.e4 (2008).

34 Ashton, A. W. \& Ware, J. A. Thromboxane A2 receptor signaling inhibits vascular endothelial growth factor-induced endothelial cell differentiation and migration. Circ. Res. 95, 372-379 (2004).

35 Parra, E. J., Marcini, A., Akey, J., Martinson, J., Batzer, M. A., Cooper, R. et al. Estimating African American admixture proportions by use of population-specific alleles 63, 1839-1851 (1998).

36 Adler, N.E. \& Rehkopf, D. H. US disparities in health: descriptions, causes, and mechanisms. Ann. Rev. Public Health 29, 235-252 (2008).

37 Tian, C., Plenge, R. M., Ransom, M., Lee, A., Villoslada, P., Selmi, C. et al. Analysis and application of European genetic substructure using $300 \mathrm{~K}$ SNP information. PLoS Genet. 4, e4 (2008).

38 Mackenbach, J. P., Stirbu, I., Roskam, A.- J. R., Schaap, M. M., Menvielle, G., Leinsalu, M. et al. Socioeconomic inequalities in health in 22 European countries. N. Engl. J. Med. 358, 2468-2481 (2008).

39 Yamaguchi-Kabata, Y., Nakazono, K., Takahashi, A., Saito, S., Hosono, N., Kubo, M. et al. Japanese population structure, based on SNP genotypes from 7003 individuals compared to other ethnic groups: effects on population-based association studies. Am. J. Hum. Genet. 83, 445-456 (2008).

40 Imada, Y., Fujimoto, M., Hirata, K., Hirota, T., Suzuki, Y., Saito, H. et al. Large scale genotyping study for asthma in the Japanese population. BMC Res. Notes 2, 54 (2009).

41 Kagamimori, S., Gaina, A. \& Nasermoaddeli, A. Socioeconomic status and health in the Japanese population. Soc. Sci. Med. 68, 2152-2160 (2009).

Supplementary Information accompanies the paper on Journal of Human Genetics website (http://www.nature.com/jhg) 